\title{
Adaptive Flower Pollination Algorithm: A Novel Approach for Function Optimization Problem
}

\author{
Tahsin Aziz \\ Ahsanullah University \\ of Science and Technology \\ Dhaka-1208, Bangladesh
}

\author{
Md. Rashedul \\ Karim Chowdhury \\ Ahsanullah University \\ of Science and Technology \\ Dhaka-1208, Bangladesh
}

\author{
Md. Ahsanul Bari \\ Ahsanullah University \\ of Science and Technology \\ Dhaka-1208, Bangladesh
}

\author{
Mohammad \\ Shafiul Alam \\ Ahsanullah University \\ of Science and Technology \\ Dhaka-1208, Bangladesh
}

\begin{abstract}
Nature is a great source of inspiration for research tasks. Flower Pollination Algorithm is inspired by the pollination process between different plants. Pollination is a natural process between different flowering plants. By this process plants create their offspring. In the process of pollination in flowers the pollen or gamete of male flower transfers to female flower. This paper has proposed a new variant of Flower Pollination Algorithm for continuous problem on Global Optimization by using Probability Modification.
\end{abstract}

\section{General Terms}

Metaheuristics,Algorithms,Swarm Intelligence,Genetic Algorithm

\section{Keywords}

Bioinformatics, Flower Pollination Algorithm, Probability, Self Adaptive

\section{INTRODUCTION}

Swarm Intelligence (SI) concept is practiced in Artificial Intelligence(AI). It is the unified characteristic of distributed, self-funded systems, familiar or fabricated.

SI procedures can be found generally of a population of simple samples communicating with one another and with their surroundings. The components of biological systems follow very general and simple rules but there is no specific structure that how an individual sample should react. They show various characteristics some are local and some are random. These various types of communication between different individuals interactions between such agents lead to the evolution of global characteristics. Some examples of swarm intelligence process in nature systems is ant colonies, artificial bee colony, bird flocking, hawks hunting, bacterial growth, fish schooling etc.

A Genetic Algorithm (GA) is a Meta-heuristic algorithm. This algorithm is influenced by the natural selection process. To generate high-quality solutions by depending on bio-inspired operators such as mutation, crossover and selection; Genetic Algorithm is used for optimization. By reflecting the process of natural selection, that is the fittest individuals are selected for recreation in order to produce child of the next generation [1] [2] [6].

Flower Pollination Algorithm is combined by Swarm Intelligence Algorithm and Genetic Algorithms. The algorithm is based on the pollination process of flowering plants. Flower pollination is an intriguing process in the natural world. Its evolutionary characteristics leads to design new optimization algorithms.
This paper has tried to improve the value of probability by customizing it by using self-adaptive formula. Then it compares the performance of the Original Flower Pollination Algorithm with the new algorithm which is developed by changing the probability value.

The rest of this paper is organized as follows. Section 2 describes the Flower Pollination Algorithm. Section 3 provides details of the self-adaptive probability value. Section 4 gives the simulation and analysis of this modified algorithm with the original algorithm on the benchmark problems, parameter settings of the algorithms and compares their results. Finally, section 5 draws the closure of this paper with a few comments and suggestions on future research.

\section{FLOWER POLLINATION ALGORITHM}

\subsection{Characteristics of Flower Pollination}

Plants grow from seeds. Plants use flowers to recreate their offsprings. This process depends on the seed. Seeds are produced if pollen is transferred between the same species of flower. Flower pollination is typically associated with the transfer of pollen via pollinators or vectors such as insects, birds, bats and other animals [9] [?].

Pollination occurs by the result of an animal's activity on a flower. When the pollinator collects food from a flower the pollen grains of that flower are attached with the pollinator. If it visits another flower for the same reason then the pollen can be drop off and can occur a successful pollination [?].

Pollination can take two major forms: Abiotic and Biotic [?] [3] [4]. In Biotic pollination, pollen is carried to the stigma by insects and animals and this can be happened in long distances. On the other hand, in Abiotic pollination, pollination occurs via wind or diffusion in water [?].

About ninty percent of the pollination process of flowering plants belongs to Biotic pollination where the vectors carry pollen. Ten percent of pollination takes Abiotic form which does not require any pollinators.

Pollination can be achieved by either of self-pollination or cross-pollination. Self-pollination means pollen from a plant's stamen is transferred to that same plant's stigma whereas Crosspollination means pollen from a plant's stamen is transferred to a different plant's stigma [?] [9].

Flower constancy is maintained by visiting exclusive certain flower species while bypassing other flower species. In addition, bees and birds may behave as Lévy flight behaviour with jump or fly distance steps obey a Lévy distribution. 


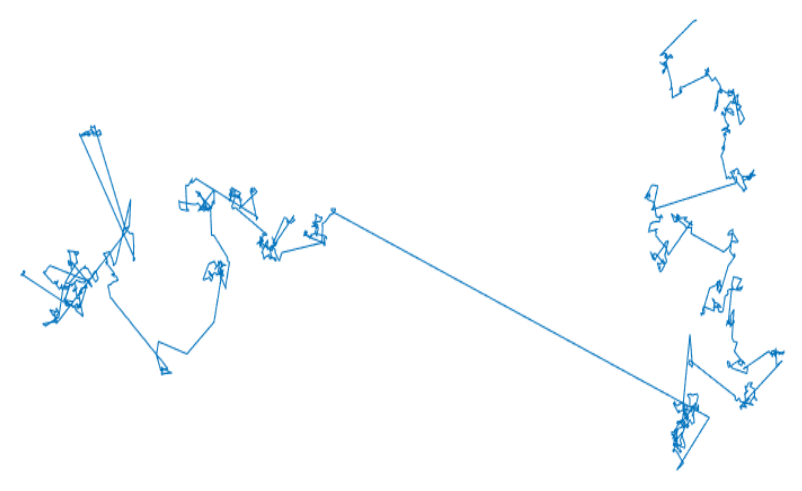

Fig. 1: Lévy Flight

Xin She Yang describes this flower constancy and pollinator behavior in the pollination process into the following four rules:

(1) Biotic and cross-pollination is considered as global pollination process with pollen-carrying pollinators performing Lévy flights.

(2) Abiotic and self-pollination are considered as local pollination.

(3) Flower constancy can be considered as the reproduction probability is proportional to the similarity of two flowers involved.

(4) Local pollination and global pollination is controlled by a switch probability $p \in[0,1]$. Besides the physical proximity and other factors like wind and water, local pollination can have a significant fraction $p$ in the overall pollination process.

\subsection{Global Pollination and Local Pollination}

The two key steps in Flower Pollination Algorithm are: Global Pollination Process and Local Pollination Process [?]. In global pollination process, flower pollens are carried by pollinators so they may travel over a long distance because pollinators can often fly and move in longer range. This global pollination can be represented mathematically as

$$
x_{i}^{t+1}=x_{i}^{t}+\gamma L(\lambda)\left(x_{i}^{t}-g_{*}\right)
$$

Here $x_{i}^{t}$ is the pollen $i$ or solution vector $x_{i}$ at iteration $t$, and $g^{*}$ is the current best solution found among all solutions at the current iteration. Here $\lambda$ is a scaling factor to control the step size. Hence, parameter $L(\lambda)$ is also the step size which corresponds to the strength of the pollination [?] [?] [?]. Since pollinators can be travelled over a long distance with different distance steps. Here,
Lévy flight can be used to mirror this travelling characteristic. Assuming $L>0$ from a Lévy distribution

$$
L \sim \frac{\lambda \Gamma(\lambda) \sin (\pi \lambda / 2)}{\pi} \frac{1}{s^{1+\lambda}}, \quad\left(S \gg S_{0}>0\right)
$$

Here, $\Gamma(\lambda)$ is the standard gamma function and Lévy distribution is valid for long steps $S>0$. Therefore, Rule 2 and Rule 3 which are basically for the local pollination can be represented like

$$
x_{i}^{t+1}=x_{i}^{t}+\epsilon\left(x_{j}^{t}+x_{k}^{t}\right)
$$

Here, $x_{j}^{t}$ and $x_{k}^{t}$ are pollen from different flowers of the same plant species. The equation narrates flower constancy in limited neighborhoods [?]. Assuming in mathematically if $x_{j}^{t}$ and $x_{k}^{t}$ comes from the same species or selected from the same population, this equivalently becomes a local random walk if a graph can be drawn $\epsilon$ from a uniform distribution in $[0,1]$. The pseudo code of the flower pollination algorithm is given below.

\subsection{Pseudo Code of Flower Pollination Algorithm}

In reality every plant can have multiple flowers and each flower patch can release millions and billions of pollen gametes. However, to eliminate the complexity, Xin-She Yang assumed that each plant has only one flower and each flower only produce one gamete.So there is no necessity to distinguish between a pollen gamete or a flower or a plant.

For the simplicity, one pollen gamete is characterised by $x_{i}$. The most fittest solution is $g_{*}$.

In Algorithm 1 we have described the FPA Algorithm in details.

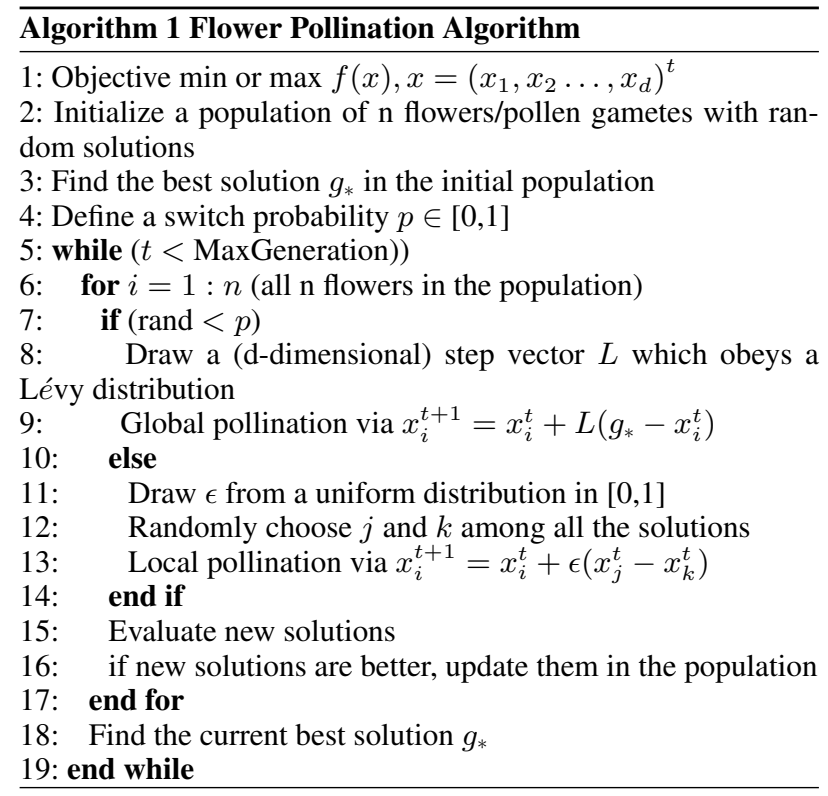

\section{MODIFIED FLOWER POLLINATION ALGORITHM}

\subsection{Modification of Switch Probability}

In Flower Pollination Algorithm, the Biotic and CrossPollination is considered as Global Pollination whereas Abiotic 
and Self-Pollination is considered as Local Pollination.

In the algorithm it can be seen that the Switch Probability controls the global and local pollination. In the original algorithm the value of switch probability $p$ was declared as constant and the value is 0.8 , which means in most of the time this algorithm does global pollination.

This paper has tried to modify this value of $p$ depending on the fitness of the pollen after some iterations. To change the value of $p$ learning strategies are used. The concepts for learning strategies are taken from Self Adaptive Differential Evolution Algorithm for Numerical Optimization.

To probabilistic-ally select one out of several available learning strategies and use it on current population is the idea of using Learning Strategy Adaptation.

In this paper the improved algorithm has initially set the value of $p$ as 0.8 and the value is updated by using customized Self-Adaptive Techniques after every fifty iterations.

\section{SIMULATION AND ANALYSIS}

\subsection{Benchmark Functions}

To compare between the Original Flower Pollination Algorithm with the new modified version, this paper has taken help from benchmark functions. A set of four benchmark functions suit is used by this paper. In the suit some of the functions are uni-modal where as some of the functions are multi-modal. A uni-modal function has only one local optimum whereas multi-modal has multiple local optima. The search process must be able to avoid getting trapped at regions around local minima to reach global minima in multimodal functions. It also consists of high dimensional and low dimensional optimization functions.

This paper runs a test whether the results have been improved or not. The analytical form of each function, along with their names and bounds of search space of the functions are shown in Table 1. For all the functions the global minimum value $f_{\min }$ is 0.0 . The benchmark functions that we will be using are as follows:

\subsection{Parameter Settings}

Both algorithms are tested with 100 independent runs on each of the test functions listed in Table 1 The swarm size is set to 25 . The number of generations is set to 1000,1500 and 2000 for D $=10,30$ and 50 respectively for each run. The initial probability value $p$ is set as 0.8 in the simulation. Matlab version R2016a is used for the simulation.

\subsection{Experimental Results}

In Swarm Intelligence Algorithm, there should be a balance between the degrees of explorations and exploitations with which the swarm individuals search across the search space. Too much exploitation may cause the algorithm to be captured around the locally optimal points making difficult or even impossible to find the global optimum. In the contrary, too much exploration slows down the convergence speed and compact the overall search performance.

Flower Pollination Algorithm controls the degrees of explorations and exploitations with a switch probability $p$. In FPA the global and local pollination technique is used to balance between explorations and exploitations. The Lévy distribution is applied to generate new solutions in Global Pollination while in local pollination new solutions are generated using randomly selected local solutions. The Lévy distribution has the capability to generate new solutions with bigger mutation step size enabling the algorithm to escape from the locally optimal points.

From the experimental results, it can be seen that the proposed method shows greater result in some of the cases than Original Flower Pollination Algorithm.

For simulation, four benchmark functions have been used in this experiment. Among them two functions are uni-modal and two are multi-modal. In Table 2 , the comparison between the two algorithms can be observed.

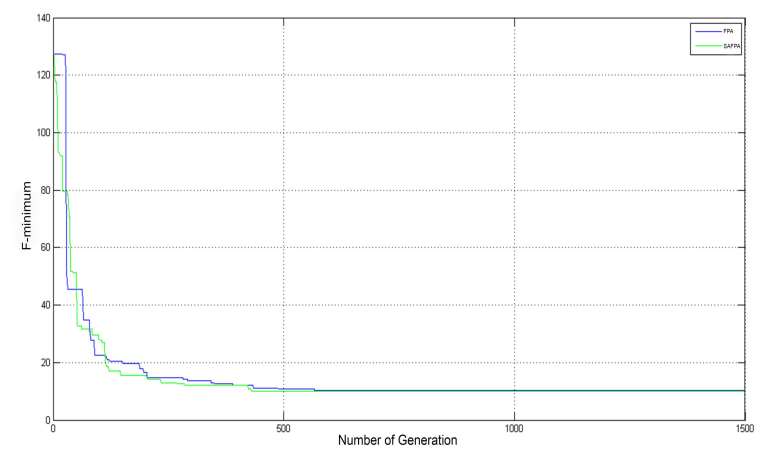

Fig. 2: Graphical representation for 1500 iterations for Quartic Function

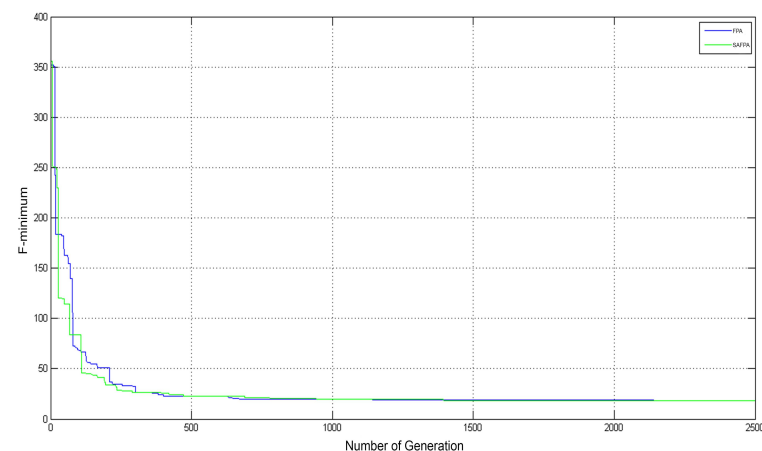

Fig. 3: Graphical representation for 2500 iterations for Quartic Function

For Uni-modal Functions, the comparison shows that the proposed method has showed better result for Quartic Function but at the same time the result for Zakharov Function has not been that much satisfactory.

For Multi-modal Functions, the comparison shows that the proposed method has showed better result for both functions.

Figure 2 and Figure 3 shows graphical representation of $f_{\min }$ vs generations for dimension 10 and dimension 30 . 


\section{CONCLUSION}

This paper proposes a method to update the value of switch probability in self adaptation process. Flower Pollination Algorithm uses swarm intelligence to find the global optimum value of the continuous optimization problems. Numerical results on the standard benchmark problems for the algorithms demonstrate the effectiveness and competitiveness of the algorithms based on the value of $p$. Few research works try to improve the performance of the Flower Pollination algorithm. In future we plan to compare with those algorithms with the Flower Pollination algorithm with modified switch probability by using learning strategies.

\section{REFERENCES}

[1] Y. Xin-She, "Engineering Optimization: An Introduction with Metaheuristic Application," Wiley, 2010.

[2] H. A. Abbass and R. Sarker, "The Pareto diffential evolution algorithm,' Int. J. Artificial Intelligence Tools, vol. 11(4), pp. 531-552, 2002.

[3] K. S, "Pollination based optimization," in 6th International Multi Conference on Intelligent Systems, Sustainable, New and Renewable Energy Technology and Nanotechnology (IISN2012), March 16-18,2012.

[4] N. Waser, "Flower constancy: definition, cause and measurement," The American Naturalist, 1986.

[5] A.-R. Osama, A.-B. Mohamed and E.-h. Ibrahim, "A Novel Hybrid Flower Pollination Algorithm with Chaotic Harmony Search for Solving Sudoku Puzzles," International Journal of Engineering Trends and Technology (IJETT), vol. 7, no. 3, pp. 126-132, January 2014.

[6] Y. Xin-She,"Nature-inspired Metaheuristic Algorithms," Luniver Press, 2008

[7] Y. Xin-She, K. Mehmet and H. Xingshi, "Multi-objective Flower Algorithm for Optimization," in International Conference on Computational Science, ICCS 2013, 2013.

[8] Y. Xin-She, "Flower pollination algorithm for global optimization," Unconventional Computation and Natural Computation, Lecture Notes in Computer Science, vol. 7445, p. 240249, 2012.

[9] M. Walker, "How flowers conquered the world," BBC Earth News, 10 July 2009.

[10] K. Gaganpreet and D. S. Dr., "Pollination Based Optimization or Color Image Segmentation," International Journal of Computer Engineering and Technology (IJCET), vol. 3, no. 2, pp. 407-414, July-September 2012. 
Table 1. : Benchmark functions used in the experimental studies. Here, D: Dimensionality of the function, S: search space, C: function characteristics with values $\mathrm{U}$ : Uni-modal and M: Multi-modal.For all the function $f_{\min }=0.0$

\begin{tabular}{|c|c|c|c|c|c|}
\hline Function No & Function Name & $\boldsymbol{D}$ & $\boldsymbol{C}$ & $\boldsymbol{S}$ & Function Definition \\
\hline$f_{1}$ & Schwefel & $10,30,50$ & $\mathrm{M}$ & {$[-500,500]^{D}$} & $f_{1}(x)=418.9829 d-\sum_{i=1}^{d} x_{i} \sin \sqrt{\left|x_{i}\right|}$ \\
\hline$f_{2}$ & Quartic & $10,30,50$ & $\mathrm{U}$ & {$[-1.28,1.28]^{D}$} & $f_{2}(x)=\sum_{i=0}^{n} i x_{i}^{4}$ \\
\hline$f_{3}$ & Zakharov & $10,30,50$ & $\mathrm{U}$ & {$[-5,10]^{D}$} & $f_{3}(x)=\sum_{i=1}^{d} x_{i}{ }^{2}+\left(\sum_{i=1}^{d} 0.5 i x_{i}\right)^{2}+\left(\sum_{i=1}^{d} 0.5 i x_{i}\right)^{4}$ \\
\hline$f_{4}$ & Michalewicz & $10,30,50$ & $\mathrm{M}$ & {$[0, \pi]^{D}$} & $f_{4}(x)=-\sum_{i=1}^{d} \sin \left(x_{i}\right) \sin ^{2 m}\left(\frac{x_{i}^{2}}{\pi}\right)$ \\
\hline
\end{tabular}

Table 2. : Comparison of FPA and SAFPA on four standard benchmark functions. Algorithms are run 100 different times on each of the functions. The result of SAFPA for each dimensionality is marked with boldface font.

\begin{tabular}{|c|c|c|c|c|c|c|c|c|}
\hline Function Number & Function Name & Algorithm & Dimension & Best & Worst & Mean & Median & $S D$ \\
\hline \multirow{6}{*}{1} & \multirow{6}{*}{ Schwefel } & FPA & \multirow[b]{2}{*}{10} & $5.05 \mathrm{E}+02$ & $1.37 \mathrm{E}+03$ & $1.02 \mathrm{E}+03$ & $1.03 \mathrm{E}+03$ & $1.47 \mathrm{E}+02$ \\
\hline & & SAFPA & & $6.63 \mathrm{E}+00$ & $1.38 \mathrm{E}+03$ & $9.94 \mathrm{E}+02$ & $1.04 \mathrm{E}+03$ & $2.54 \mathrm{E}+02$ \\
\hline & & FPA & \multirow[b]{2}{*}{30} & $3.86 \mathrm{E}+03$ & $5.47 \mathrm{E}+03$ & $4.92 \mathrm{E}+03$ & $4.95 \mathrm{E}+03$ & $2.80 \mathrm{E}+02$ \\
\hline & & SAFPA & & $3.65 \mathrm{E}+03$ & $5.83 \mathrm{E}+03$ & $5.03 E+03$ & $5.00 \mathrm{E}+03$ & $3.33 \mathrm{E}+02$ \\
\hline & & FPA & \multirow[b]{2}{*}{50} & $7.18 \mathrm{E}+03$ & $9.89 \mathrm{E}+03$ & $9.02 \mathrm{E}+03$ & $9.07 \mathrm{E}+03$ & $4.19 \mathrm{E}+02$ \\
\hline & & SAFPA & & $6.46 \mathrm{E}+03$ & $1.01 \mathrm{E}+04$ & $9.03 E+03$ & $9.08 \mathrm{E}+03$ & $5.42 \mathrm{E}+02$ \\
\hline \multirow{6}{*}{2} & \multirow{6}{*}{ Quartic } & FPA & \multirow[b]{2}{*}{10} & $1.35 \mathrm{E}+00$ & $2.61 \mathrm{E}+00$ & $2.13 \mathrm{E}+00$ & $2.17 \mathrm{E}+00$ & $2.68 \mathrm{E}-01$ \\
\hline & & SAFPA & & $1.42 \mathrm{E}+00$ & $2.63 \mathrm{E}+00$ & $2.13 E+00$ & $2.16 \mathrm{E}+00$ & $2.38 \mathrm{E}-01$ \\
\hline & & FPA & \multirow{2}{*}{30} & $8.38 \mathrm{E}+00$ & $1.14 \mathrm{E}+01$ & $1.02 \mathrm{E}+01$ & $1.02 \mathrm{E}+01$ & $5.76 \mathrm{E}-01$ \\
\hline & & SAFPA & & $8.16 \mathrm{E}+00$ & $1.17 \mathrm{E}+01$ & $1.02 \mathrm{E}+01$ & $1.03 \mathrm{E}+01$ & $5.71 \mathrm{E}-01$ \\
\hline & & FPA & \multirow{2}{*}{50} & $1.59 \mathrm{E}+01$ & $2.18 \mathrm{E}+01$ & $1.92 \mathrm{E}+01$ & $1.93 \mathrm{E}+01$ & $8.66 \mathrm{E}-01$ \\
\hline & & SAFPA & & $1.64 \mathrm{E}+01$ & $2.12 \mathrm{E}+01$ & $1.91 \mathrm{E}+01$ & $1.92 \mathrm{E}+01$ & $8.90 \mathrm{E}-01$ \\
\hline \multirow{6}{*}{3} & \multirow{6}{*}{ Zakharov } & FPA & \multirow{2}{*}{10} & $9.79 \mathrm{E}-05$ & $4.13 \mathrm{E}-03$ & $9.13 \mathrm{E}-04$ & $7.23 \mathrm{E}-04$ & $7.18 \mathrm{E}-04$ \\
\hline & & SAFPA & & $1.47 \mathrm{E}-02$ & $1.90 \mathrm{E}+01$ & 8.96E-01 & $3.06 \mathrm{E}-01$ & $2.14 \mathrm{E}+00$ \\
\hline & & FPA & \multirow[b]{2}{*}{30} & $5.66 \mathrm{E}+00$ & $1.01 \mathrm{E}+02$ & $4.62 \mathrm{E}+01$ & $4.26 \mathrm{E}+01$ & $1.89 \mathrm{E}+01$ \\
\hline & & SAFPA & & $3.45 \mathrm{E}+01$ & $1.88 \mathrm{E}+02$ & $9.16 \mathrm{E}+01$ & $8.76 \mathrm{E}+01$ & $2.91 \mathrm{E}+01$ \\
\hline & & FPA & \multirow[b]{2}{*}{50} & $7.31 \mathrm{E}+01$ & $2.69 \mathrm{E}+02$ & $1.42 \mathrm{E}+02$ & $1.37 \mathrm{E}+02$ & $3.98 \mathrm{E}+01$ \\
\hline & & SAFPA & & $1.13 \mathrm{E}+02$ & $5.54 \mathrm{E}+02$ & $2.35 \mathrm{E}+02$ & $2.27 \mathrm{E}+02$ & $6.46 \mathrm{E}+01$ \\
\hline \multirow{6}{*}{4} & \multirow{6}{*}{ Michalewicz } & FPA & \multirow[b]{2}{*}{10} & $-7.76 \mathrm{E}+00$ & $-6.17 \mathrm{E}+00$ & $-6.97 \mathrm{E}+00$ & $-6.99 \mathrm{E}+00$ & $3.62 \mathrm{E}-01$ \\
\hline & & SAFPA & & $-8.02 \mathrm{E}+00$ & $-5.81 \mathrm{E}+00$ & $-6.79 \mathrm{E}+00$ & $-6.76 \mathrm{E}+00$ & $4.03 \mathrm{E}-01$ \\
\hline & & FPA & \multirow[b]{2}{*}{30} & $-1.74 \mathrm{E}+01$ & $-1.30 \mathrm{E}+01$ & $-1.45 \mathrm{E}+01$ & $-1.44 \mathrm{E}+01$ & $8.24 \mathrm{E}-01$ \\
\hline & & SAFPA & & $-1.69 \mathrm{E}+01$ & $-1.24 \mathrm{E}+01$ & $-1.43 \mathrm{E}+01$ & $-1.44 \mathrm{E}+01$ & $8.50 \mathrm{E}-01$ \\
\hline & & FPA & \multirow[b]{2}{*}{50} & $-2.43 \mathrm{E}+01$ & $-1.83 \mathrm{E}+01$ & $-2.08 \mathrm{E}+01$ & $-2.07 \mathrm{E}+01$ & $1.33 \mathrm{E}+00$ \\
\hline & & SAFPA & & $-2.48 \mathrm{E}+01$ & $-1.69 \mathrm{E}+01$ & $-2.05 \mathrm{E}+01$ & $-2.04 \mathrm{E}+01$ & $1.60 \mathrm{E}+00$ \\
\hline
\end{tabular}

